\title{
Raman characterization and stress analysis of AIN grown on SiC by sublimation
}

L. Liu, B. Liu, and J. H. Edgara)

Department of Chemical Engineering, Kansas State University, Manhattan, Kansas 66506

S. Rajasingam and M. Kuball

H. H. Wills Physics Laboratory, University of Bristol, Bristol BS8 1TL, United Kingdom

(Received 5 April 2002; accepted 17 July 2002)

\begin{abstract}
The stress distribution in bulk AIN crystals seeded on $6 \mathrm{H}-\mathrm{SiC}$ was theoretically modeled and also determined experimentally from Raman peak positions. The full width at half maximum of the AlN Raman peaks showed the crystal quality improved as its thickness increased. The theoretical frequency shifts of the $E_{1}$ (transverse optical) mode calculated from model-predicted stress were in good agreement with experimental values taken along the edges of crystal samples. The stress was linearly distributed along the depth of the samples, and changed from compressive at the growing surface to tensile at the interface between $\mathrm{AlN}$ and $\mathrm{SiC}$ for thickness range of several hundred micrometers. Large tensile stresses, up to $0.6 \mathrm{GPa}$, were detected in the AlN at the interface. The effects of growth temperature and sample thickness were investigated. It is predicted that the AlN on $6 \mathrm{H}-\mathrm{SiC}$ must be at least $2 \mathrm{~mm}$ thick to prevent it from cracking while cooling down the sample from a growth temperature of $2000^{\circ} \mathrm{C}$. (C) 2002 American Institute of Physics.
\end{abstract}

[DOI: $10.1063 / 1.1506195]$

\section{INTRODUCTION}

Recently group-III nitrides have attracted much interest due to their enormous potential for electronic and optoelectronic device applications. The lack of native bulk substrates for epitaxy is a major limitation for fully implementing such devices. Compared to the substrates now used commercially, mainly sapphire and $\mathrm{SiC}$, AlN exhibits superior properties that are expected to enhance the performance of III-nitride devices. ${ }^{1}$ In particular, AlN substrates would have good chemical compatibility, lattice/crystal structure match, thermal expansion match, high thermal conductivity, high electrical resistivity, and excellent oxidation resistance. In addition, AlN is also considered as one of the best materials for fabrication of surface acoustic wave devices. ${ }^{2}$

Most studies of AlN bulk crystal growth have employed the sublimation method. This technique was most successfully developed by Slack and McNelly ${ }^{3,4}$ in the mid 1970s. Large boule (diameter up to $15 \mathrm{~mm}$ ) crystals have been produced by self seeding in sealed tungsten crucibles with a sharp tip. ${ }^{5} 6 \mathrm{H}-\mathrm{SiC}$ substrates offer an alternative method of seeding even larger AlN crystals by sublimation due to the easy AlN nucleation and crystal orientation/polarity control. $^{6-9}$

Previously, we have grown AlN by sublimation using a semisealed tungsten crucible in a resistively heated tungsten furnace and investigated the effect of substrate preparation on the sublimation growth of AlN at about $1800^{\circ} \mathrm{C}$ and 400 Torr on $(0001) 6 \mathrm{H}-\mathrm{SiC}{ }^{10}$ Three problems with using $6 \mathrm{H}-$ $\mathrm{SiC}$ as the seed crystals were identified: the decomposition of

\footnotetext{
a) Author to whom correspondence should be addressed; electronic mail: chemail@cheme.ksu.edu
}

$\mathrm{SiC}$ at high growth temperature; three-dimensional (3D) nucleation and consequent formation of individual grains in the AlN crystal; and cracking of the AlN crystal due to the thermal expansion coefficients mismatch between AlN and $\mathrm{SiC}$. To circumvent the first two problems, an AlN epitaxial layer was first deposited on $\mathrm{SiC}$ substrates by metalorganic chemical vapor deposition (MOCVD), resulting in 2D growth mode and suppressing $\mathrm{SiC}$ decomposition and grain boundary formation. ${ }^{10,11}$

This work addresses the last problem: the stress distribution in the AlN crystals grown on $\mathrm{SiC}$ substrates. In this work, a theoretical model was formulated to describe the stress distribution in these $\mathrm{AlN} / 6 \mathrm{H}-\mathrm{SiC}$ heterostructures. The Raman shift measurements were taken along the crosssectional edge of several samples at $20 \mu \mathrm{m}$ intervals. Applying the known relationship between the Raman shift and stress, the stress distributions in AlN samples were determined from shifted Raman frequencies and compared to the model-predicted ones.

\section{EXPERIMENT}

\section{A. Crystal growth}

AlN buffer layers were first deposited on $\mathrm{Si}$ polarity (0001) 6H-SiC substrates in a vertical MOCVD induction heated reactor. Trimethylaluminum (TMA) and ammonia $\left(\mathrm{NH}_{3}\right)$ were utilized as $\mathrm{Al}$ and $\mathrm{N}$ sources, and $\mathrm{Pd}$ cell purified $\mathrm{H}_{2}$ was the carrier gas. The buffer layers were deposited at $1000^{\circ} \mathrm{C}$ and 80 Torr under $\mathrm{H}_{2}, \mathrm{NH}_{3}$, and TMA flow rates of $6 \mathrm{slm}, 3 \mathrm{slm}$, and $60 \mathrm{sccm}$, respectively. An approximate $1.5 \mu \mathrm{m}$ thick AlN film was obtained for a $2 \mathrm{~h}$ run. These AlN buffered $6 \mathrm{H}-\mathrm{SiC}$ crystals were then used as substrates for AlN sublimation. 
The experimental setup for the AlN sublimation growth was described in detail in previous work. ${ }^{10,12}$ Briefly, the Al and $\mathrm{N}_{2}$ vapor created by the dissociation of the hot polycrystalline AlN source $\left(T_{s} \sim 1900{ }^{\circ} \mathrm{C}\right)$ was transported to a seed held at a lower temperature $\left(T_{c} \sim 1800{ }^{\circ} \mathrm{C}\right)$ in a tungsten crucible. Then adsorption/desorption took place at the surface of the growing $\mathrm{AlN}$ on $6 \mathrm{H}-\mathrm{SiC}$ substrates leading to crystal growth. The sublimation was carried out in an ultrahigh purity $\mathrm{N}_{2}$ atmosphere of 400 Torr. The temperature gradient was maintained by a resistively heated tungsten element and the pressure was automatically maintained at a constant value. Sintered AlN with approximate $0.6 \mathrm{wt} \%$ oxygen as the main impurity (as analyzed by a standard inert gas fusion method) was the source material. AlN crystals were grown for several tens of hours, producing AlN layers greater than $200 \mu \mathrm{m}$ thick.

\section{B. Characterization}

Raman scattering spectroscopy provides a noninvasive method for measuring the stress in heterostructures with spatial resolution as small as $1 \mu \mathrm{m}$. Micro-Raman spectra were obtained using a Dilor XY micro-Raman system with the 514 nm line of an Ar-laser as the excitation source. The crystal was placed on its edge, so that the wurtzite $c$ axis is perpendicular to the incident light. Raman light was measured through a polarizer to select the Raman light of perpendicular polarization to the incident light. The experiment is thus in $x(y z)-x$ orientation, i.e., light incident in the $x$ direction, polarized in the $y$ direction, Raman light was measured in the $-x$ direction, polarized in the $z$ direction (where the $z$ direction is the wurtzite $c$ axis). Note that in this orientation, the $E_{1}$ transverse optical (TO) mode is excited uniquely. The physical size of the laser spot at optimum focus is about 1-2 $\mu \mathrm{m}$. The measured Raman light is excited from this region. It can be used as a rough measure of spatial resolution. The spectral resolution is a limit of accuracy of the spectrometer itself, and is $0.1 \mathrm{~cm}^{-1}$. Raman spectra were taken at $20 \mu \mathrm{m}$ intervals along the edge of the cleaved AlN film/6H-SiC cross sections. The intervals were set by physically moving the microscope stage and measuring the distance of the laser spot from the top surface.

\section{THEORY}

\section{A. Stress model formulation}

Due to the high sublimation temperature and difference in thermal expansion coefficients, large stresses are introduced into both the AlN film and the SiC substrate on cooling to room temperature in the $c$ plane. Thus it is important to understand the temperature and thickness dependence of stress and the minimal thickness to prevent cracks from forming in the AlN deposited on SiC substrates.

We assume there is negligible AIN deposition when the temperature is initially increased in the furnace. Stress is only introduced due to the mismatch of thermal expansion when the composite $\mathrm{AlN} / 6 \mathrm{H}-\mathrm{SiC}$ is cooled to room temperature. ${ }^{13}$ The lattice mismatch stress is relieved during the initial growth by the incorporation of defects such as threading dislocations in the crystal. On cooling, the thermal

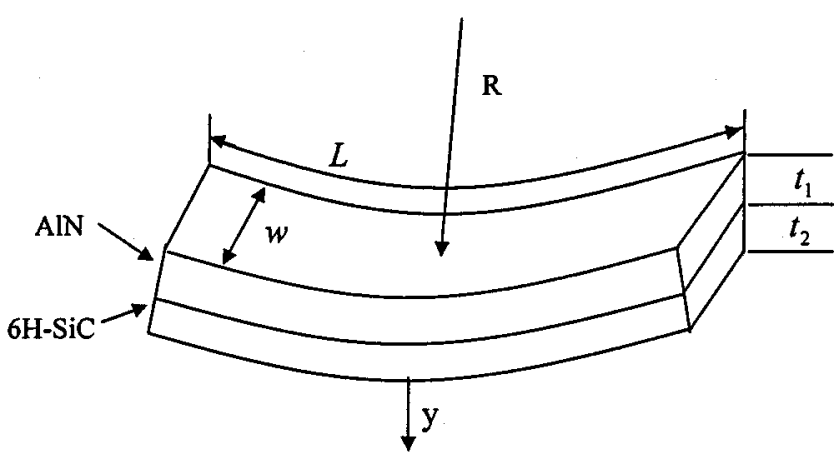

FIG. 1. Sketch of AlN/6H-SiC structure after cooling down.

contraction of $\mathrm{AlN}$ is greater than that of $\mathrm{SiC}$, so the structure bends toward the AlN when it cools down, as depicted in Fig. 1, where $R$ is the radius of curvature, $L$ is the length, $W$ is the width, $t$ is the thickness, and $y$ is the depth into the sample from the growing surface.

AlN has the wurtzite structure, thus four of the six independent elastic constants $C_{i j}$ are needed to fully describe the in-plane stress $\left(\sigma_{x x}\right)$ and normal stress $\left(\sigma_{z z}\right)$ along the $c$ plane. ${ }^{14}$ However, there is considerable variation in the literature values of these constants; e.g., $-C_{13} /\left(C_{11}+C_{12}\right)$ relating $\varepsilon_{x x}$ and $\varepsilon_{z z}$ for biaxial stress $\left(\sigma_{z z}=0\right)$ known as Poisson's effect, ${ }^{14}$ varies by a factor of $1.7 .{ }^{15}$ Note that thermal stresses in the epitaxial layers are biaxial by nature. ${ }^{13}$ In contrast, the isotropic elastic constants $E$ can be determined within only moderate uncertainties. For many purposes, it is convenient to assume isotropic elastic modulus. ${ }^{16}$ This assumption was adopted here using elastic moduli values of 329.7 and 444.0 GPa for AlN and SiC, respectively. ${ }^{17}$ The effect of the temperature on the elastic moduli was ignored.

The stress distribution for heteroepitaxial structures can be formulated using the following equations for force balance, momentum balance, and equal lattice constants at the interface. ${ }^{18}$ There are a total of $(N+1)$ equations for the variables: the force $F_{i}$ in each layer $i$ and the radius of curvature $R$. In our case, $N=2$ for an AlN on the SiC structure

$$
\begin{aligned}
& \sum_{i=1}^{N} F_{i}=0 \\
& \sum_{i=1}^{N}\left[\frac{E_{i} t_{i}^{3} w}{12 R}+F_{i} \sum_{j=1}^{i}\left(t_{j}-\frac{1}{2} t_{i}\right)\right]=0 \\
& \sum_{i=1}^{N-1}\left(\varepsilon_{i}-\varepsilon_{i+1}\right)=\frac{F_{i+1}}{E_{i+1} w t_{i+1}}-\frac{F_{i}}{E_{i} w t_{i}}-\frac{1}{2 R}\left(t_{i}+t_{i+1}\right)
\end{aligned}
$$

The thermal strain is expressed as

$$
\varepsilon_{i}=\left(\frac{a_{l}-a_{h}}{a_{h}}\right)_{i}=\int_{h}^{l} \alpha_{i} d T,
$$

where the lattice parameter at high (indicated by subscript $h$ ) or low (indicated by subscript $l$ ) temperature is obtained by the temperature-dependent thermal expansion coefficient $a$, 
TABLE I. Room temperature Raman-active phonon frequencies $\left(\mathrm{cm}^{-1}\right)$ of AlN grown by different methods.

\begin{tabular}{lccc}
\hline \hline Symmetry & $\begin{array}{c}\text { Values from } \\
\text { Ref. 21 }\end{array}$ & $\begin{array}{c}\text { Crystal on AlN } \\
\text { buffered 6H-SiC }\end{array}$ & $\begin{array}{c}\text { Crystal on as- } \\
\text { received 6H-SiC }\end{array}$ \\
\hline$E_{2}$ (low) & $\ldots$ & $\ldots$ & $\ldots$ \\
$E_{2}($ high $)$ & 657 & 652 & 653 \\
$A_{1}(\mathrm{TO})$ & 610 & $\ldots$ & $\ldots$ \\
$A_{1}(\mathrm{LO})$ & 890 & 892 & 891 \\
$E_{1}(\mathrm{TO})$ & 670 & $\ldots$ & $\ldots$ \\
$E_{1}(\mathrm{LO})$ & 912 & $\ldots$ & $\ldots$ \\
\hline \hline
\end{tabular}

which is described by the multifrequency Einstein model. ${ }^{19}$ This first principle theory can predict lattice parameters to $0.01 \%$.

Strictly speaking, to calculate the stress used in Eq. (3) requires the actual lattice constants at the interface at the growth temperature- that is the strained values of the lattice constants in the composite structure, as opposed to unconstrained crystals. For simplification, the entire strain at room temperature was assumed to be only due to the change in lattice constants with temperature-that is the strain is calculated from Eq. (4). Our simulation shows only a small error $(<2 \%)$ was introduced by directly using thermal strain in Eq. (4).

The thickness variation is also a factor for stress besides the force itself as expressed by two terms in Eq. (5).

$$
\sigma_{y}=\frac{F_{i}}{t_{i} w}+\frac{E}{R}\left(y-\frac{t_{i}}{2}\right) .
$$

If cracking occurs, the stress at the $1-2$ (i.e. $\mathrm{SiC}-\mathrm{AlN}$ ) interface $\left(y=t_{1}\right)$ should be equal to the critical cracking (tensile) stress $\sigma_{c}$

$$
\sigma_{c}=\frac{F_{1}}{t_{1} w}+\frac{E t_{1}}{2 R} .
$$

No measurements of the critical tensile stress $\sigma_{c}$ for pure AlN crystals have been reported. A value of $266 \mathrm{MPa}$ taken from the reported value for polycrystal ceramic AlN was used in this work. ${ }^{20}$

\section{B. Relationship between Raman frequency shift and stress}

A complete first-principles calculation of the phonon deformation potentials in wurtzite AlN was given in Ref. 14. In the range of the validity of Hooke's law, the magnitude of the frequency shift for each phonon mode $\lambda=A_{1}$ longitudinal optical (LO) and TO, $E_{1}$ (LO and TO), and $E_{2}$ (high and low) is determined by the two deformation potential constant $a_{\lambda}$ and $b_{\lambda}$ in the case of given strain, or by $\widetilde{a}_{\lambda}$ and $\widetilde{b}_{\lambda}$ for given stress, respectively,

$$
\Delta \omega_{\lambda}=2 a_{\lambda} \varepsilon_{x x}+b_{\lambda} \varepsilon_{z z}=2 \widetilde{a}_{\lambda} \sigma_{x x}+\tilde{b}_{\lambda} \sigma_{z z} .
$$

In this work, the $E_{1}(\mathrm{TO})$ mode (nominally at $\left.670 \mathrm{~cm}^{-1}\right)^{21}$ was used to detect the stresses on the $c$ plane of AlN since there is no $\mathrm{SiC}$ mode near it. In this case, the value $\widetilde{a}_{\lambda}$ equals $-1.29 \mathrm{~cm}^{-1} / \mathrm{GPa}$.
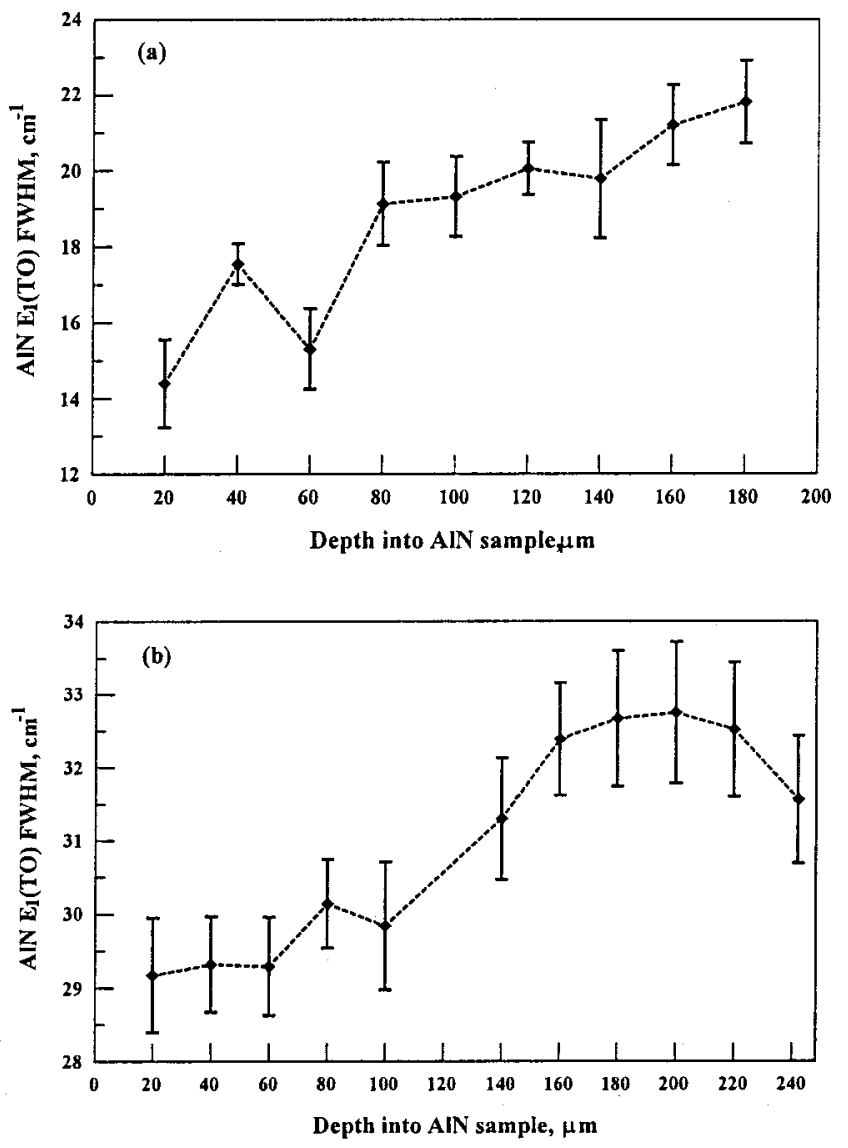

FIG. 2. FWHM profile of $E_{1}(\mathrm{TO})$ frequencies of AlN grown on: (a) asreceived $6 \mathrm{H}-\mathrm{SiC}$ (b) $6 \mathrm{H}-\mathrm{SiC}$ with AlN epilayer.

\section{RESULTS AND DISCUSSION}

\section{A. Raman data}

In wurtzite AlN, group theory predicts eight sets of phonon modes: $2 E_{2}, 2 A_{1}, 2 E_{1}$ and $2 B_{1}$ of which both $E_{2}$ sets, one $A_{1}$ set and one $E_{1}$ set are Raman active, while the $B_{1}$ modes are silent. ${ }^{22}$ Due to the different quality/impurity level/stress of the various AlN films or bulk crystals, there are some mismatches for those mode positions. ${ }^{23}$ Table I shows Raman frequencies of unstressed $\mathrm{AlN}^{21}$ together with those measured at the AlN sample surface grown by different methods in this work at room temperature.

Table I shows that the Raman peak positions are essentially the same for AlN grown on AlN buffered or as received $6 \mathrm{H}-\mathrm{SiC}$ substrates at the same growth condition and growth time, although there is significant growth-mode difference for these two substrates. ${ }^{10}$ Stress during growth appeared to be relieved by the introduced threading dislocations and was independent of the growth mode. Full width half maximums (FWHMs) of $E_{1}$ (TO) frequencies for AlN grown on as received $6 \mathrm{H}-\mathrm{SiC}$ and $6 \mathrm{H}-\mathrm{SiC}$ with the AlN epilayer decrease with increasing thickness, as illustrated in Fig. 2, indicating that the crystal quality improves with the AlN layer thickness. Figure 3 shows typical Raman signals for AlN grown on $6 \mathrm{H}-\mathrm{SiC}$ recorded on the front and back surface of the sample in the $z(x)-z$ polarization. 


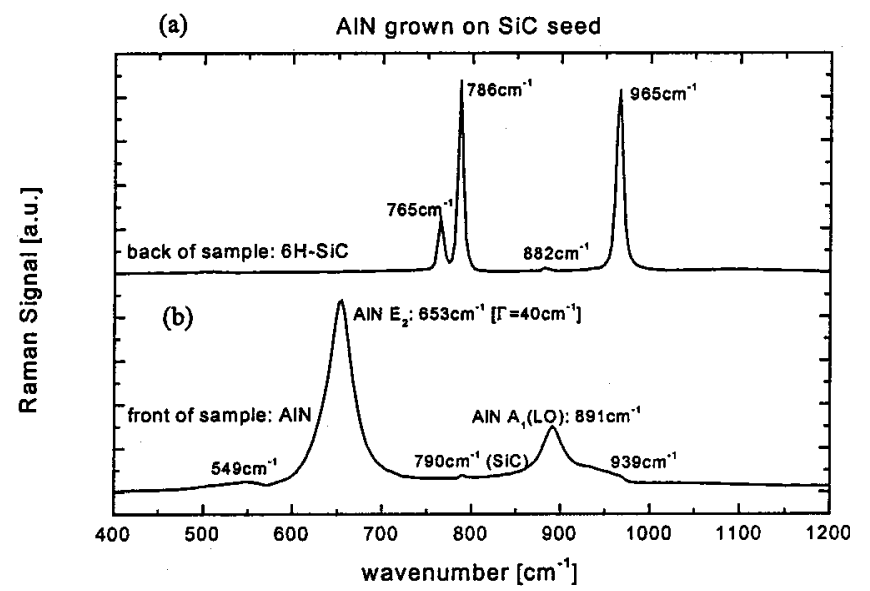

FIG. 3. A typical Raman spectra for: (a) $6 \mathrm{H}-\mathrm{SiC}$ and (b) $\mathrm{AlN}$ on $6 \mathrm{H}-\mathrm{SiC}$.

\section{B. Stress analysis}

Figures 4 and 5 present measured AlN $E_{1}(\mathrm{TO})$ Raman position and corresponding biaxial stress determined using Eq. (7) along the sample depth, where depth $=0$ means surface. The Raman frequency position for stress-free bulk AlN (grown by free nucleation) is $669.7 \mathrm{~cm}^{-1}$. The frequency shifts are positive at the growing surface and negative at the interface with $\mathrm{SiC}$. This results from the compressive stress (negative) at the surface and tensile stress (positive) at the interface, a consequence of AlN's larger thermal expansion coefficient than SiCs, and cooling from the growth temperature to room temperature. In addition, the frequency shift and stress distribution along the sample edge were approximately linear. There is a slight flattening of stress near the surface of the sample depicted in Fig. 5, whose cause still remains unknown.

Applying the stress model developed above, the stress distributions on the two samples investigated by Raman were determined, from which the theoretical Raman positions were obtained. Figures 6 and 7 compare the experimental stress distribution with the theoretical one. The results for both AlN samples deposited AlN buffered $6 \mathrm{H}-\mathrm{SiC}$ and asreceived $6 \mathrm{H}-\mathrm{SiC}$ reveal that the model prediction agrees

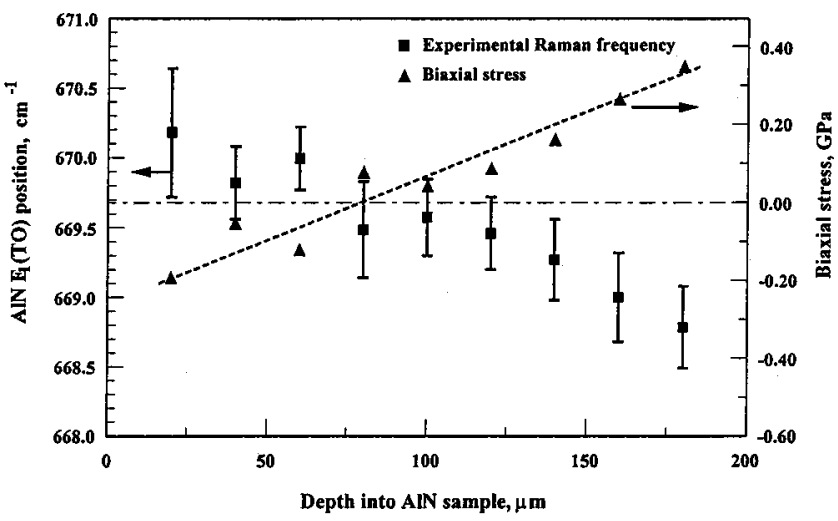

FIG. 4. Measured position of the AlN $E_{1}(\mathrm{TO})$ Raman mode and calculated biaxial stress along the sample depth with AlN thickness $187 \mu \mathrm{m}$ and residual $\mathrm{SiC}$ thickness $90 \mu \mathrm{m}$ cooling down from $1780^{\circ} \mathrm{C}$.

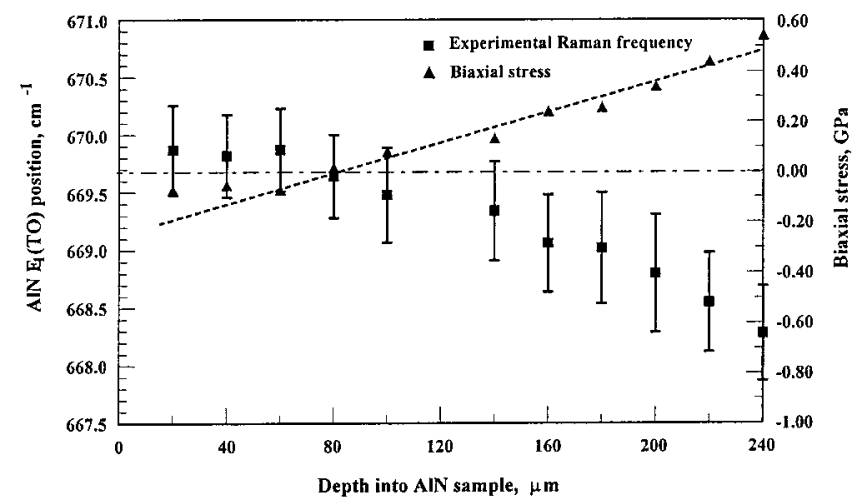

FIG. 5. Measured position of the AlN $E_{1}$ (TO) Raman mode and calculated biaxial stress along the sample depth with AlN thickness $258 \mu \mathrm{m}$ and residual $\mathrm{SiC}$ thickness $32 \mu \mathrm{m}$ cooling down from $1820^{\circ} \mathrm{C}$.

well with the experimental results. This illustrates that the approximations made in the model are justified.

\section{Stress distribution simulations}

The simulated stress distribution in AlN/SiC heterostructures as functions of film and substrate thicknesses and growth temperatures are displayed in Figs. 8 and 9. Positive values correspond to tensile stresses and negative values correspond to compressive stresses. The surface of the AlN layer corresponds to the free surface upon which growth occurs. The surface of the $\mathrm{SiC}$ corresponds to the backside of the $\mathrm{SiC}$ substrate, opposite of where AlN growth takes place - that is, it is the carbon face of the $\mathrm{SiC}$ substrate.

The dependence of the stress at the top free AlN surface, the surface (backside) of the $\mathrm{SiC}$, and the $\mathrm{AlN} / \mathrm{SiC}$ interface (in both the AlN and the $\mathrm{SiC}$ ) on the AlN layer thickness is displayed in Fig. 8. At the AlN/SiC interface, the AlN is under tensile stress and the $\mathrm{SiC}$ is under compressive stress for the entire AlN layer thickness considered (up to 10000 $\mu \mathrm{m})$. For an AlN layer thickness less than roughly $50 \mu \mathrm{m}$, the stress in the AlN at both the surface and the interface are the same sign; both are under tension. For thicker AlN layers, the stress has opposite signs in the AlN at the surface and

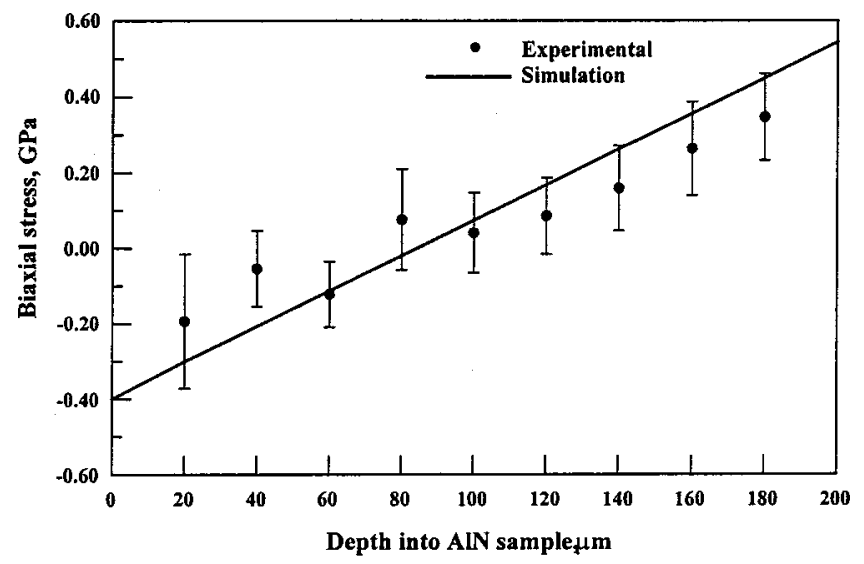

FIG. 6. Comparison of experimental and theoretical biaxial stress along the sample depth with AlN thickness $187 \mu \mathrm{m}$ deposited on AlN buffered SiC and residual $\mathrm{SiC}$ thickness $90 \mu \mathrm{m}$ cooling down from $1780^{\circ} \mathrm{C}$. 


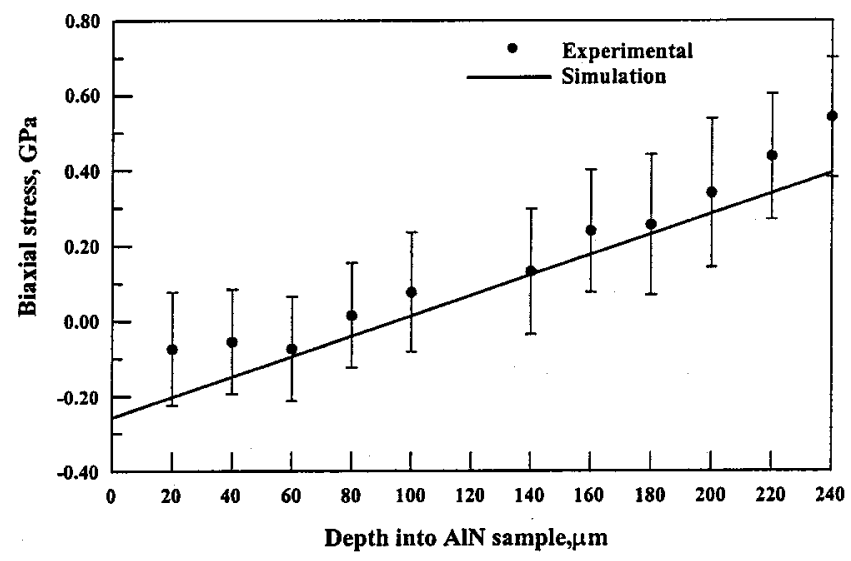

FIG. 7. Comparison of experimental and theoretical biaxial stress along the sample depth with AlN thickness $258 \mu \mathrm{m}$ deposited on as-received $\mathrm{SiC}$ and residual $\mathrm{SiC}$ thickness $32 \mu \mathrm{m}$ cooling down from $1820^{\circ} \mathrm{C}$.

interface; the interface is still under tension but the surface is under compression. In addition, the magnitude of the stress at both positions in the AlN does not vary monotonically with the AlN layer thickness, but exhibits extrema.

Figure 9(a) shows the dependence of the stress at the AlN surface and the AlN/SiC interface with the AlN layer thickness for two growth temperatures, 2000 and $1600{ }^{\circ} \mathrm{C}$. As might be expected, the larger temperature change (that is, cooling from $2000^{\circ} \mathrm{C}$ ) introduces a larger stress in the AlN. For example, for a $100 \mu \mathrm{m}$ thick AlN layer, at the AlN/SiC interface the tensile stress is $0.1 \mathrm{GPa}$ higher for the sample cooled from $2000{ }^{\circ} \mathrm{C}$ than for the sample grown at $1600{ }^{\circ} \mathrm{C}$. Figure 9(b) shows the dependence of the stress at the AlN surface and the AlN/SiC interface with the AlN layer thickness for two residual $\mathrm{SiC}$ substrate thicknesses, at a fixed growth temperature of $1800{ }^{\circ} \mathrm{C}$. The two curves have similar shapes and magnitudes, but the curve shifts to a higher AlN layer thickness as the residual $\mathrm{SiC}$ thickness is increased. The minimum stress at the AlN surface occurs at $500 \mu \mathrm{m}$ for the thick residual $\mathrm{SiC}$, compared to $100 \mu \mathrm{m}$ for the thinner residual $\mathrm{SiC}$.

To prevent cracking, the stress in the AlN must not exceed its critical cracking stress of $266 \mathrm{MPa}$. This can be

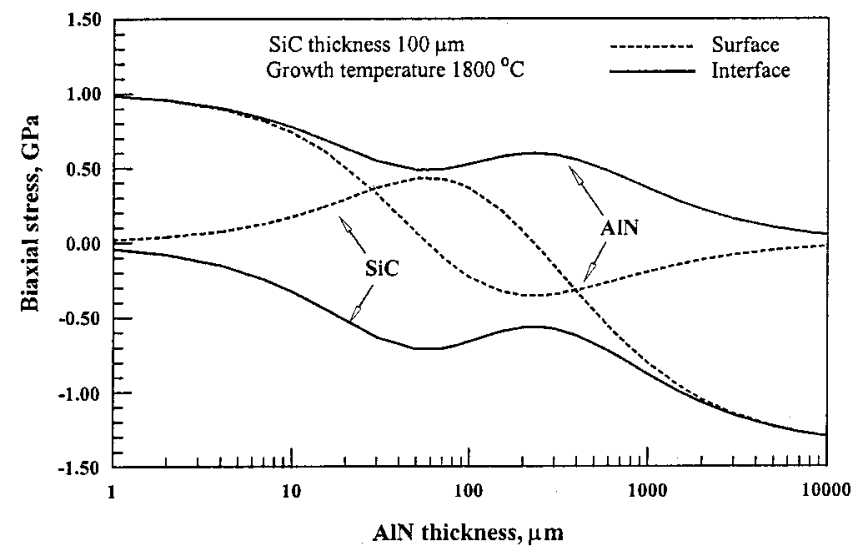

FIG. 8. The dependence of stress at surface and interface of the AlN-SiC layers on AlN thickness for a growth temperature of $1800{ }^{\circ} \mathrm{C}$, a postgrowth temperature of $20^{\circ} \mathrm{C}$, and $\mathrm{SiC}$ thickness of $100 \mu \mathrm{m}$.
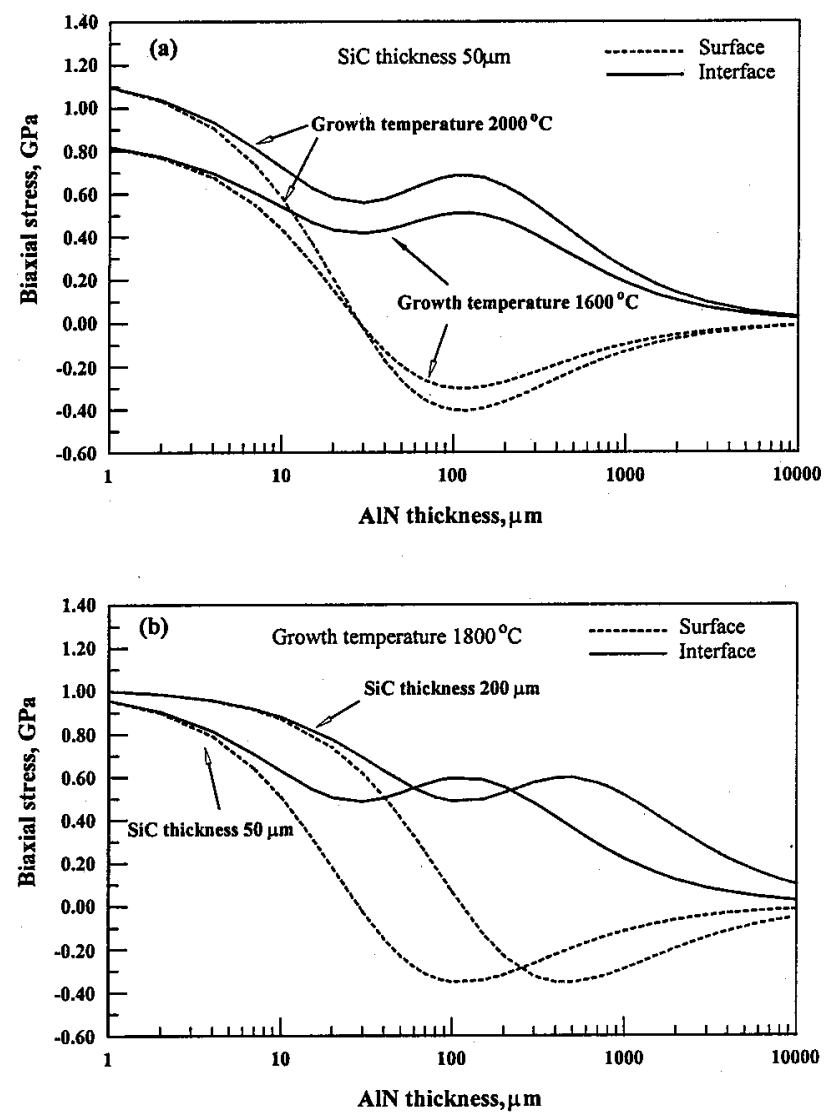

FIG. 9. The dependence of stress distribution in AlN layer on: (a) growth temperature and (b) residual $\mathrm{SiC}$ thickness.

achieved by increasing the AlN thickness to reduce the stress at the interface. Below this thickness, the AlN interface suffers a stress greater than the critical value and hence cracking occurs. From Fig. 8, we predict that the deposited AlN layer thickness must be larger than $2 \mathrm{~mm}$ to eliminate the cracks for the residual $\mathrm{SiC}$ substrate thickness of $100 \mu \mathrm{m}$ for the growth temperature of $1800{ }^{\circ} \mathrm{C}$. From Fig. 9, it is obvious that this AlN critical thickness increases with the increase of growth temperature or residual $\mathrm{SiC}$ thickness. Of course, this is approximate due to the simplifying assumptions made in the model. In addition, there is a limitation that this model cannot predict cracking density.

\section{SUMMARY AND CONCLUSIONS}

Cracks due to the lattice and mainly large expansion coefficient mismatch were observed in AlN crystals deposited on $6 \mathrm{H}-\mathrm{SiC}$ substrates. A theoretical model was presented to calculate the stress distribution in the heterostructure of AlN grown on $6 \mathrm{H}-\mathrm{SiC}$ based on the thickness and the growth temperature. The stress introduced during the growth is negligible compared to the stress created by the thermal expansion mismatch during cooling down. The presence of an AlN buffer layer deposited by MOCVD does not change stress distribution. Good agreement was found between the theoretically and experimentally determined stress distributions in the AlN grown on $\mathrm{SiC}$ as function of sample depth. It was estimated the AlN must be that at least $2 \mathrm{~mm}$ to 
prevent cracking in the AlN layer, due to its low critical cracking stress at such high growth temperature.

\section{ACKNOWLEDGMENTS}

Kansas State University acknowledges the financial support of the Office of Naval Research through Grant Nos. N00014-97-1-0332 and N00014-99-1-0104. Bristol University acknowledges financial support from EPSRC through Grant No. GR/R02207/01.

${ }^{1}$ B. Monemar, J. Mater. Sci.: Mater. Electron. 10, 227 (1999).

${ }^{2}$ O. Ambacher, J. Phys. D 31, 2653 (1998).

${ }^{3}$ G. A. Slack and T. F. McNelly, J. Cryst. Growth 34, 263 (1976).

${ }^{4}$ G. A. Slack and T. F. McNelly, J. Cryst. Growth 42, 560 (1977).

${ }^{5}$ J. C. Rojo, G. A. Slack, K. Morgan, L. J. Schowalter, and M. Dudley, Mater. Res. Soc. Symp. Proc. 639, G1.10 (2001).

${ }^{6}$ C. M. Balkas, Z. Sitar, T. Zheleva, L. Bergman, R. Nemanich, and R. F. Davis, J. Cryst. Growth 179, 363 (1997).

${ }^{7}$ W. L. Sarney, L. Salamanca-Riba, T. Hossain, P. Zhou, H. N. Jayatirtha, H. H. Kang, R. D. Vispute, M. Spencer, and K. A. Jones, MRS Internet J. Nitride Semicond. Res. 5S1, W5.5 (2000).

${ }^{8}$ S. Yu. Karpov, D. V. Zimina, Yu. N. Makarov, E. N. Mokhov, A. D. Roenkov, M. G. Ramm, and Yu. A. Vodakov, Phys. Status Solidi A 176, 435 (1999).

${ }^{9}$ S. Segal, S. Yu. Karpov, Yu. N. Makarov, E. N. Mokhov, A. D. Roenkov, M. G. Ramm, and Yu. A. Vodakov, J. Cryst. Growth 211, 68 (2000).
${ }^{10}$ L. Liu, B. Liu, Y. Shi, and J. H. Edgar, MRS Internet J. Nitride Semicond. Res. 6, 7 (2001).

${ }^{11}$ Y. Shi, Z. Y. Xie, L. Liu, B. Liu, J. H. Edgar, and M. Kuball, J. Cryst. Growth 233, 177 (2001).

${ }^{12}$ L. Liu and J. H. Edgar, J. Cryst. Growth 220, 243 (2000).

${ }^{13}$ C. Kisielowski, in Gallium Nitride II, edited by J. I. Pankove and T. D. Moustakas (Academic, London, 1999).

${ }^{14}$ J. M. Wagner and F. Bechstedt, Appl. Phys. Lett. 77, 346 (2000).

${ }^{15}$ R. R. Reeber and K. Wang, MRS Internet J. Nitride Semicond. Res. 6, 3 (2001).

${ }^{16}$ C. Kisielowski, J. Kruger, S. Ruvimov, T. Suski, J. W. Ager III, E. Jones, Z. Liliental-Weber, M. Rubin, and E. R. Weber, Phys. Rev. B 54, 17745 (1996).

${ }^{17}$ K. Wang and R. R. Reeber, MRS Internet J. Nitride Semicond. Res. 4S1, G3.18 (1999).

${ }^{18}$ K. Hiramatsu, T. Detchprohm, and I. Akasaki, Jpn. J. Appl. Phys., Part 1 32, 1528 (1993)

${ }^{19}$ R. R. Reeber and K. Wang, Mater. Res. Soc. Symp. Proc. 622, T6.35.1 (2000).

${ }^{20}$ J. F. Shackelford and W. Alexander, Material Science and Engineering Handbook, 3rd ed. (Chemical Rubber Corp., Boca Raton, FL, 2001), p. 808.

${ }^{21}$ J. M. Hayes, M. Kuball, Y. Shi, and J. H. Edgar, Jpn. J. Appl. Phys., Part 2 39, L710 (2000).

${ }^{22}$ M. Kuball, Surf. Interface Anal. 31, 987 (2001).

${ }^{23}$ A. Yoshida, in Properties, Processing and Applications of Gallium Nitride and Related Semiconductors, edited by J. H. Edgar, S. Strite, I. Akasaki, H. Amano, and C. Wetzel (Inspec, London, 1999). 